\title{
Configuración de identidad racial de aprendientes de segundas lenguas en el contexto colombiano
}

\author{
Configuration of \\ Racial Identity in \\ Second Language \\ Learners in the \\ Colombian Context
}

Configuração da identidade racial de estudantes de segunda língua no contexto colombiano

Ferney Cruz Arcila* https://orcid.org/0000-0002-9185-9392

Sandra Ximena Bonilla Medina** https://orcid.org/0000-0002-6625-501X

\section{Para citar este artículo}

Cruz Arcila, F. y Bonilla Medina, S. (2021).

Configuración de identidad racial de aprendientes de segundas lenguas en el contexto colombiano. Folios, (54). https:// doi.org/10.17227/folios.54-11330

* Doctor en Lenguaje, Discurso y Comunicación del King's College, Londres. Profesor del Instituto Caro y Cuervo, Bogotá, Colombia.

Correo: ferney.cruz@caroycuervo.gov.co

** Doctora en Educación University of East London. Profesora de la Universidad Distrital Francisco José de Caldas, Bogotá, Colombia.

Correo: sxbonillam@udistrital.edu.co 


\title{
Resumen
}

Este artículo de investigación presenta los hallazgos de un estudio orientado a explorar el tipo de conexiones que pueden existir entre el aprendizaje de segundas lenguas y la configuración de identidades raciales de aprendientes de español y de inglés. Aunque este tipo de conexiones tiene grandes implicaciones para las prácticas de enseñanza y aprendizaje de lenguas en nuestro país, se ha dado por sentado y ha permanecido inexplorado. Apoyados en la teoría critica de la raza -en particular, en el concepto de blancura-, además de postulados poscoloniales, la investigación buscó problematizar la noción de identidad racial al analizarla como un factor relevante en procesos de aprendizaje de lenguas. Mediante una metodología cualitativa, el análisis de la construcción de identidades raciales se hizo por medio de narrativas escritas y orales. Entre los hallazgos principales se destaca la idea de que el aprendizaje de lenguas está directamente relacionado con formas de desafío de estructuras raciales en algunas ocasiones y de acomodación a estas, en otras. Dichos desafíos y acomodaciones se ven reflejados en diferentes tipos de posicionamientos ante diversos grupos sociales por parte de los aprendientes.

\section{Palabras clave}

raza; identidad racial; aprendizaje de lenguas; aprendientes de segundas lenguas

\begin{abstract}
This research article discusses the findings of a study aimed at exploring the type of connections that may exist between second language learning and the configuration of racial identities of Spanish and English learners. Despite its great implications for language teaching and learning in our country, this type of connections has been taken for granted and has remained unexplored. This is how this research sought to problematize the notion of racial identity by analyzing it as a relevant factor in language learning processes. Following a qualitative methodology, the analysis of the construction of racial identities was done through written and oral narratives. Among the main findings, the study highlights the idea that language learning is directly related to ways to either challenge or accommodate racial structures. These challenges and accommodations are reflected in different types of learners' positionings towards different social groups.

\section{Keywords}

race; racial identity; language learning; second language learners

Este artigo apresenta as descobertas de um estudo que visa explorar os tipos de conexões que podem existir entre a aprendizagem de segundas línguas e a configuração de identidades raciais de estudantes de espanhol e de inglês. Embora estas conexões tenham grandes implicações para as práticas de ensino e aprendizagem de línguas em nosso país, tem-se dado como certa a falta de necessidade de exploração do tema. Assim foi como a investigação buscou problematizar a noção de identidade racial ao analisá-la como um fator relevante em processos de aprendizagem de línguas. Mediante uma metodologia qualitativa, a análise da construção de identidades raciais se fez por meio de narrativas escritas e orais. Dentro das principais descobertas se destaca a ideia de que a aprendizagem de línguas está diretamente relacionada com as formas de desafio de estruturas raciais em algumas ocasiões e de acomodação às mesmas, em outras. Tais desafios e acomodações estão refletidos em diferentes tipos de posicionamento diante de diversos grupos sociais por parte dos estudantes.
\end{abstract}

Abstract

\section{Resumo}

\section{Palavras chave}

raça; identidade racial; aprendizagem de línguas; estudantes de segunda língua 


\section{Introducción}

Hoy, la idea de que el lenguaje es constitutivo de la identidad de los individuos es irrefutable (Morgan, 2004; Norton, 2013). Incluso hay quienes, desde una postura posestructuralista, sostienen que la construcción de identidad es un proceso netamente discursivo (Davies y Harré, 1990; Harré et al., 2009; De Fina y Georgakopoulou, 2012). Es decir, mediante el uso del lenguaje en interacción con otros es posible posicionarnos de una manera u otra. En el campo del aprendizaje de lenguas, como lo explica Norton (2013), esto quiere decir que,

[...] cada vez que los aprendientes de idiomas hablan, leen o escriben en la lengua meta, no solo están intercambiando información con miembros de la comunidad de este idioma, sino que también están organizando y reorganizando el sentido de quiénes son y cómo se relacionan con el mundo social. En otras palabras, están involucrados en la construcción y negociación de identidades. (p. 4, traducción propia)

Como lo indica Norton, entonces, el aprendizaje de segundas lenguas necesariamente implica nuevos sentidos sobre las percepciones que los aprendientes tienen o pueden tener sobre quiénes son. Esto a su vez implica que haya tensiones sobre la idea de quiénes son los usuarios de determinadas lenguas o lo que puede significar convertirse en usuarios de una de ellas (cf. Anya, 2017).

En este contexto, una premisa de la que partió el estudio es que dichas tensiones y nuevos significados también están muy ligados a la forma en que los aprendientes se identifican o son identificados racialmente. La lengua es un factor identificador de grupos étnicos y culturales y, por ende, un elemento que podría reproducir, pero también combatir, configuraciones y divisiones raciales. Aunque no exclusivamente, las lenguas indígenas, por ejemplo, podrían ser consideradas como un elemento constitutivo de las personas que se identifican como indígenas. Igualmente, lenguas como el español o el inglés y sus variedades tienden a ser relacionadas directamente con diferencias raciales entre comunidades de habla e incluso acríticamente podrían ser tomadas como punto de referencia para establecer divisiones raciales (p. ej., se tiende a pensar que el español de América es el español de mestizos, y el español de España es el español de blancos europeos). Por tanto, las ideas que se tengan sobre determinados grupos raciales tienden a ser reproducidas en las actitudes hacia determinadas lenguas y sus usuarios.

A pesar de la importancia que reviste la conexión entre identidad racial y aprendizaje de lenguas con respecto a las prácticas y políticas educativas, este es un asunto que aún no ha sido investigado a profundidad (Kubota y Lin, 2009). Es cierto que, tomando un enfoque netamente lingüístico, ha surgido una corriente teórica denominada raciolinguistics (lingüística racial), que de manera muy acertada se ocupa de estudiar la relación entre lengua, raza y poder (Anderson, 2015; Alim, Rickford y Ball, 2016; Rosa y Flores, 2017). Sin embargo, los procesos de aprendizaje de lenguas no han sido centro de análisis. En relación directa con el aprendizaje de lenguas, los estudios existentes han tendido a explorar las implicaciones identitarias raciales en el aprendizaje de lenguas de comunidades indígenas en América al aprender español (Dubeau, 2011; Valencia-Galvis, 2012) o la manera en que comunidades afros se ven representadas en materiales de enseñanza (Agudelo, 2007; Clavijo, 2017; Cuba-Vega, 2014; Lefèvre y Okome, 2015), o en las tensiones que se generan para comunidades inmigrantes, cuya lengua se ve supeditada y limitada a las normas de la comunidad dominante (p. ej., Arreguín-Anderson y Ruiz-Escalante, 2014; Redondo-Quesada, 2018).

La investigación existente ha tendido a limitar la exploración de asuntos de raza y aprendizaje de lenguas a grupos que tradicionalmente son categorizados y clasificados por sus rasgos fenotípicos o procedencia sociocultural. Por ejemplo, sobresalen categorías como indígenas, afrodescendientes e inmigrantes. Con respecto a los primeros, por ejemplo, se ha dicho que su identidad está muy ligada a las lenguas nativas y que, por consiguiente, las motivaciones para aprender una segunda lengua son netamente instrumentales, como ampliar capitales 
económicos y culturales o, simplemente, combatir la discriminación (Debeau, 2011; Valencia-Galvis, 2012). En cuanto a comunidades afrodescendientes, algunas investigaciones han mostrado que, en materiales de enseñanza de lenguas, estas son "mal-visibilizadas" ya que casi nunca se tienen en cuenta $y$, cuando aparecen, se refuerzan muchos estereotipos o ideas exotistas (Cuba-Vega, 2014; Lefèvre y Okome, 2015; Soler, 2006). De otra parte, se ha analizado cómo, al estar en contacto con grupos lingüísticos y culturales mayoritarios, los inmigrantes han sido objeto de políticas restrictivas, estratificaciones y diferentes tipos de conflictos identitarios (Arreguín y Ruiz-Escalante, 2014; RedondoQuesada, 2018; Usma, Medina y Ortiz, 2018).

Si bien investigaciones existentes han desvelado importantes conexiones entre cuestiones de raza e identidad, se ha dejado de lado la idea de que existen implicaciones identitarias raciales con todos los aprendientes, sin importar sus antecedentes étnicos. Al parecer, en los estudios previos, se ha ignorado el hecho de que las comunidades reconocidas como mayoritarias (p. ej., blancos europeos) también se encuentran constituidas identitariamente en unas estructuras raciales que podrían estar relacionadas con el aprendizaje de segundas lenguas. A partir de lo anterior, y teniendo como punto de referencia que la raza es un constructo social, este estudio se centró en los siguientes interrogantes: ¿Cómo se relacionan los procesos de aprendizaje del español e inglés como segundas lenguas en el contexto colombiano con la construcción de identidad racial de los aprendientes de estas lenguas? ¿Qué relaciones se pueden establecer entre los significados que los aprendientes otorgan al aprendizaje de una segunda lengua en particular y la construcción de sus identidades raciales?

Se han tomado como referentes el español y el inglés por varias razones: el inglés - concebido como la lengua de los negocios o el desarrollo económico o como la lengua franca por excelencia que favorece la comunicación intercultural- que con tanto ahínco se ha venido promoviendo en el sistema educativo nacional por medio de políticas de bilingüismo
(MEN, 2013, 2014, 2016), emergió como un punto de referencia ineludible en el objeto de estudio de esta investigación. Este trabajo pretende contribuir a entender de mejor manera la incidencia que la promoción y aprendizaje de esta lengua ha podido tener en las propias percepciones de identidades de aprendientes, al ser una lengua que se ha presentado como obligatoria. Por otro lado, considerando que el español es la lengua oficial de nuestro país y que, en los últimos años, Colombia ha venido tomando cada vez más auge como destino predilecto de muchos extranjeros para aprender español (Ministerio de Cultura, 2018), resulta relevante también analizar las percepciones e implicaciones de tipo identitario que se pueden generar en relación con grupos específicos de aprendientes.

En lo que sigue, se discuten algunas de las investigaciones en torno a la raza y aprendizaje de lenguas que se han llevado a cabo en Colombia. Luego se presentan los fundamentos teóricos del estudio, en donde se deja claro cómo algunos principios de la teoría crítica de la raza y de la poscolonialidad proporcionan un marco analítico sólido para abordar las preguntas de esta exploración. Posteriormente, se tratarán las consideraciones metodológicas que fundamentan el estudio, así como los resultados y conclusiones más relevantes.

\section{Investigación en torno a identidad racial y aprendizaje de lenguas}

El debate de la educación y la raza en relación con el aprendizaje de las lenguas extranjeras en Colombia no ha sido comúnmente explorado. Los estudios más relacionados con el campo se han realizado recientemente a raíz de los cambios derivados en la Constitución de 1991 en donde se reconoce el país como un territorio pluricultural y multiétnico. El hecho de que este campo no haya sido centro de interés investigativo ha sido evaluado por teóricos como Castro-Gómez (2000) o Mignolo (2000, 2005), como uno de los efectos del dispositivo de blancura que ha sido insertado en la dimensión cognitiva de las poblaciones originarias de territorios colonizados como Latinoamérica. Este dispositivo tiene que 
ver con la creencia de que el blanco es símbolo de superioridad no solo a nivel físico sino también intelectual y cultural (Wade, 1995). Los efectos de este dispositivo, incrustados en los emblemas políticos que definen al país como un territorio mestizo, se encargaron de esconder los intereses colonizantes y propagaron la idea de que la raza era una cuestión de tiempos de colonización y que había muerto con la independencia (Ospina, 1997; Quiceno, 2003).

A pesar de la larga temporada en que los discursos de la raza se mantuvieron apaciguados por el nuevo discurso de la igualdad mestiza, las desigualdades sociales, la inequidad y el descontento de las poblaciones afectadas por las estructuras raciales pusieron en relieve la necesidad de reactivar el tema y denunciarlo como una preocupación colectiva que reclama igualdad de derechos (Bello y Rangel, 2000; Chaves y Zambrano, 2006). Así pues, mediante una nueva disposición política constitucional, el país inicia los debates en torno a la raza con contundencia.

En el campo de la enseñanza y el aprendizaje de lenguas, los estudios que relacionan la raza, además de ser mucho más escasos, también tienden a singularizar la identidad racial en términos de mayorías y minorías. Usma, Medina y Ortiz (2018), por ejemplo, reflexionan sobre cómo las políticas lingüísticas del país, que promueven el inglés, reflejan disposiciones que en últimas desfavorecen a las poblaciones indígenas en la educación superior. Clavijo (2017), por otro lado, señala orientaciones para realizar una pedagogía basada en la comunidad que resalte los valores y principios de las comunidades indígenas, a través del aprendizaje del inglés. Asimismo, Agudelo (2007) presenta un modelo para orientar el aprendizaje de las lenguas extranjeras enfocado a incorporar el rescate de elementos socioculturales situados que permitan un balance entre el interés por el conocimiento de una cultura foránea -inmerso en el aprendizaje de la lengua extranjera- y el conocimiento de la cultura local (autóctona). Por otro lado, con relación al español como segunda lengua, Valencia-Galvis (2012) explora las maneras en que comunidades quechuas que han llegado a Medellín, negocian sus identidades culturales y lingüísticas en una metrópolis hispanohablante. El autor sostiene que, aunque factores como las transacciones comerciales y el vestuario cumplen un papel importante a la hora de mantener su identidad indígena, es la lengua quechua el elemento integrador y definitorio de su identidad, frente a los grupos mayoritarios que habitan la ciudad.

Estos estudios son ejemplos de las aproximaciones que empiezan a brotar en Colombia con respecto a la relación entre la raza, la enseñanza y el aprendizaje de lenguas extranjeras $y$, aunque muestran una tendencia por reflexionar en torno a la justicia social, como es generalmente el propósito de los estudios con este enfoque, estos reportes mantienen una perspectiva desacertada de la identidad racial al presentarla como una categoría inmutable. Desde este punto de vista, estas exploraciones incitan a pensar las identidades en transición (Thesen, 1997), ya que han emergido en las dinámicas sociales y aunque algunas veces no estén reconocidas como entidades de minorías, también se han visto afectadas por las estructuras raciales. Así mismo, analizar cómo el aprendizaje de lenguas ha incidido en la transformación de esa identidad sería una nueva dimensión que contribuya a los estudios ya iniciados en esta área.

\section{Consideraciones teóricas}

El presente estudio se basa en un concepto de la raza como construcción social y así mismo niega las explicaciones naturalistas al respecto (FlórezBolívar, 2008; McGraw, 2007; Muñoz-Gaviria y Runge-Peña, 2005; Du Bois, 2001; Fanon, 2001; Gilroy, 2004; Knowles y Alexander, 2005; Nardal, 2001). Que la raza sea una construcción social implica la premisa de que es creada y reforzada en la práctica social, es decir, no es real pero percibida como tal (Chadderton, 2009). Es importante hacer explícita esta idea ya que las etiquetas que se crean socialmente son poderosas para mantener condiciones discriminatorias contra ciertos individuos o grupos mientras que las prácticas sociales mantienen esas mismas condiciones (Banton, 2002). En este 
sentido, la raza es problematizada en este estudio para hacer un análisis por medio de las categorías raciales que, en intersección con aspectos sociales, políticos, económicos, culturales y lingüísticos coadyuvan con la inequidad, la desigualdad social y las maneras en que estas se manifiestan en procesos de aprendizaje de lenguas.

En este contexto, nos apoyamos en la teoría crítica de la raza (TCR) para abordar nuestro análisis, ya que ofrece un marco conceptual sociohistórico y crítico sobre el rol de la raza en la constitución de prácticas sociales. Como su nombre lo indica, la TCR se ha dedicado a escudriñar las estructuras sociales, económicas y culturales para cuestionar instancias en las que categorías raciales son usadas para marginar a los individuos y a los grupos sociales (Delgado y Stefancic, 2000, 2001; Ladson-Billings y Tate, 2006; Carbado y Gulati, 2003; Solorzano y Yosso, 2001). Esta teoría, creada y más popularmente reconocida en el contexto de Estados Unidos, nació en el marco legislativo a través de los intereses de los estudiosos de leyes reconocidos como de color y que pensaban que la ley no lograba dar cuenta de las experiencias de todos los individuos contribuyendo así con la discriminación. A partir del rescate a las experiencias individuales por medio de counter stories, estos investigadores lograron demostrar que dichas historias eran el reflejo de la injusticia del sistema y que debía ser ante estos parámetros de subjetividad que la justicia tendría que apostar por su interpelación.

De acuerdo con sus seguidores, la TCR ha logrado desmantelar las prácticas sociales discriminatorias del sistema que se han escondido en el sentido común y las han normalizado (Gillborn, 2010; Leonardo, 2002; Decuir y Dixson, 2004; Banks, 2002; Sleeter, 2004). En otras palabras, estas prácticas sociales discriminatorias se han vuelto aceptadas y no cuestionadas. En educación, esta teoría ha sido útil para identificar formas de discriminación que han sido intangibles a nivel macro (políticas) y que se mantienen en prácticas sociales y culturales a nivel micro (prácticas sociales) (Bonilla-Medina, 2018).

Trasladar la TCR a un contexto como el colombiano tiene la intención de vislumbrar elementos que han permitido la reproducción de prácticas discriminatorias y que muchas veces no se han entendido como tal. Sin embargo, es de apuntar que analizar el contexto colombiano desde la visión crítica de la raza tiene un riesgo: en palabras de Koopman (2012), el contexto colombiano presenta una mezcla étnica hoy en día que sería casi imposible identificar separaciones determinadas por el color de piel. En virtud de ello, y atendiendo la idea de la complejidad presentada, nos inclinamos a utilizar una derivada más contemporánea de los estudios críticos de raza que se ha encargado de teorizar este concepto desde la noción de blancura (whitenesss) (Clarke y Gardner, 2009; Bonnet, 2000; King, 1991). La blancura es una de las formas de teorización crítica de la raza que también enfatiza en la misma problemática, pero aborda el conflicto más allá del color de piel. De esta manera, la blancura no es una forma de distinguir sujetos con cierto color de piel, sino que se instala en los discursos que se han incorporado en las prácticas sociales y que han situado a algunos sujetos en una posición de desventaja a diferentes niveles sociales, económicos, políticos y culturales. Es decir, hablar de raza desde esta perspectiva implica analizar diferentes tipos de relaciones de poder determinadas por orígenes socioculturales, posicionamientos económicos, ideologías políticas, factores lingüísticos, entre muchos otros.

La línea de la blancura en los estudios críticos de la raza muestra más consonancia con el contexto colombiano debido a que este debate busca explicar las estructuras raciales fundamentadas en el concepto de su mismo nombre. La blancura es una teoría que coincide con el marcador analítico del dispositivo de blancura descrito por latinoamericanos como Castro-Gómez (2000) y Mignolo (2000; 2005). Desde una mirada de la pos-colonialidad, estos autores se han encargado de trabajar en la fundamentación de un pensamiento que arguye que los territorios latinoamericanos han sido víctimas de la colonización históricamente y que, aunque hoy en día son independientes, dicha independencia nunca se ha logrado realmente debido a que se sigue dependiendo de otros países no solo a nivel económico, sino a nivel simbólico. Argumentan 
estos autores que los intereses de colonización se mantuvieron latentes ya que las estructuras sociales, económicas y culturales ya estaban endurecidas y se convirtieron en el sentido común de los pobladores colombianos, subjetivándolos a nivel de conocimiento (colonialidad del conocimiento), a nivel político (colonialidad del poder) y a nivel del sujeto (colonialidad del ser). De tal suerte que este sentido común ha tomado forma en regímenes de verdad (Said, 1976) y ha funcionado como la plataforma para un dispositivo de blancura.

Teniendo en cuenta los argumentos presentados desde la poscolonialidad, la blancura se ha convertido en la estructura racial que algunas veces es imperceptible pero que sigue manteniendo la colonización en los pueblos aparentemente separados por movimientos independentistas. Como se dijo anteriormente, las teorías de la blancura han sido una rama de las teorías críticas de la raza que se ha enfocado en ir más allá de la discusión de color de piel como estructura racial constituida en un eje sociopolítico y económico para explorar mucho más la dimensión simbólica de la raza al asociarla con cualquier tipo de discriminación social. Así, la idea del dispositivo de blancura y el debate por un inconsciente de blancura que explican estas teorías se encuentran intersectados y pueden ser útiles para explicar lo que ocurre en el contexto de la enseñanza y aprendizaje de las lenguas en Colombia con relación a las concepciones de identidad racial, de la autoidentificación y las consecuencias que se crean mediante estas ideas.

Estudios que relacionan la identidad racial y la enseñanza y aprendizaje de lenguas extranjeras -los cuales son más populares en contextos como Estados Unidos (Thesen, 1997; Norton, 1997), Inglaterra (Leung et ál., 1997) y Australia- han mostrado mayor interés en reflexionar sobre el rol del lenguaje que en sus efectos en una identidad racial. Por eso, en este estudio, se considera que trabajar desde estas perspectivas teóricas permite un acercamiento profundo a estos temas que han permanecido inexplorados.

\section{Aproximaciones metodológicas}

Este es un estudio narrativo y, por tanto, la forma en que nos acercamos al fenómeno de interés fue por medio de narraciones de aprendientes de español y de inglés sobre sus procesos y experiencias de aprendizaje. En relación con la concepción de identidad que tomamos, las narrativas son consideradas una forma de construcción de realidad social (Somers, 1994; Spector-Mersel, 2010), ya que favorecen la creación de significados sobre fenómenos sociales (De Fina y Georgakopoulou, 2012) que se vuelven tangibles por medio de historias. La narrativa es una práctica de la naturaleza humana por medio de la cual le damos sentido al mundo en nuestro diario vivir; como lo argumenta Spector-Mersel (2010), a través de historias damos cuenta de lo que pensamos, sentimos y de cómo nos comportamos. Esin lo expresa de esta manera:

[...] las narrativas pueden verse como el vehículo a través del cual hablamos sobre nuestro mundo, nuestras vidas y nosotros mismos. Las narraciones no expresan simplemente algunas realidades individuales e independientes; más bien, ayudan a construir la realidad dentro de las relaciones entre el narrador y su mundo externo. (2011, p. 94)

Las narrativas se obtuvieron por medio de historias de vida y entrevistas a profundidad. En primera instancia, los participantes de esta investigación fueron invitados a escribir sus historias de vida con respecto a las lenguas no maternas que hablan, guiados por preguntas sobre las razones para aprenderlas, maneras en que las han aprendido, el momento en sus vidas en que las aprendieron, la importancia que tiene para ellos haberlas aprendido, experiencias alrededor del aprendizaje de dichas lenguas y los significados que asocian con estas. En segunda instancia, a partir de las historias de vida, se procedió a entrevistar a los participantes del estudio con el fin de profundizar sobre algunos de los aspectos más relevantes que, a la luz de la presente investigación, hayan compartido en las historias de vida. En términos generales, las entrevistas indagaron sobre el tipo de conexiones que los aprendientes establecen entre el sentido de quiénes 
son ellos y las lenguas que han aprendido o están aprendiendo. A partir de los cimientos teóricos discutidos anteriormente, el análisis temático fue la técnica que seguimos para interpretar los datos.

Como lo muestran las siguientes tablas 1 y 2 , el estudio contó con la participación de siete aprendientes de español y siete de inglés. Los únicos criterios de selección fueron: a) que estuvieran o hubieran vivido parte de su proceso de aprendizaje de la segunda lengua en el contexto colombiano y b) que tuvieran la disposición para participar voluntariamente. Es de anotar que el origen de los participantes le añade diversidad al análisis, especialmente teniendo en cuenta la perspectiva interseccional del concepto de raza que toma este estudio.

Tabla 1. Participantes, aprendientes de español

\begin{tabular}{|l|l|l|l|}
\hline \multicolumn{1}{|c|}{ Nombre y edad } & \multicolumn{1}{|c|}{ Procedencia } & \multicolumn{1}{c|}{ Otras lenguas } & \multicolumn{1}{c|}{ Contacto con C2 } \\
\hline Fanny (20-25) & Francia & Inglés & Colombia, Venezuela \\
\hline Mona (25-30) & India & Hindi, inglés, urdu & Colombia \\
\hline Yazmín (20-25) & China & Español (inglés) & Colombia \\
\hline Sol (30-35) & Holanda & Holandés & Bogotá \\
\hline Kato & Indígena arhuaco & Ikün & Colombia, España \\
\hline Lola (25-30) & Colombia (sorda) & Español (LSE, francés, inglés) & Colombia, España \\
\hline Liz (25-30) & Reino Unido & Francés & \\
\hline
\end{tabular}

Fuente: elaboración propia.

Como se observa, contamos con la participación de aprendientes de Francia, India, China, Holanda y el Reino Unido; de igual forma, con una aprendiente sorda y un indígena. Esta diversidad entre los participantes se vio enriquecida por los diferentes repertorios lingüísticos, por los diferentes rangos de edades y la familiaridad con diversos grupos culturales de la lengua meta.

Tabla 2. Participantes, aprendientes de inglés

\begin{tabular}{|l|l|l|l|}
\hline \multicolumn{1}{|c|}{ Nombre y edad } & \multicolumn{1}{|c|}{ Procedencia } & Otras lenguas & \multicolumn{1}{c|}{ Contacto con cultura L2 } \\
\hline Manuela (40-50) & Colombia & - & Colombia \\
\hline Charlie (25-30) & Colombia & - & Colombia, Venezuela \\
\hline Louise (20-25) & Colombia & - & Colombia \\
\hline Jhon (20-25) & Colombia & - & Colombia \\
\hline Elias (25-30) & Colombia & - & Colombia \\
\hline Cris (40-50) & Colombia & - & Reino Unido, EE. UU. \\
\hline Mía & China & $\begin{array}{l}\text { Vietnamita, mandarín, } \\
\text { inglés, español }\end{array}$ & Japón, Colombia \\
\hline & & Fuente: elaboración propia. & \\
\hline
\end{tabular}

Los aprendientes de inglés fueron en su mayoría colombianos hablantes de español como L1. Mía fue la única aprendiente de inglés extranjera cuya lengua materna es el mandarín, pero su repertorio lingüístico incluye otras tres lenguas. El grupo de aprendientes colombianos ha tenido contacto con la cultura meta (C2) principalmente desde el contexto colombiano. 
En lo que resta del artículo discutiremos los resultados más importantes a los que hemos llegado, pero antes se hace relevante explicar cómo presentamos los datos. Tomando como fuentes principales las historias de vida y las entrevistas, cuando incluimos fragmentos de los datos registramos el nombre (seudónimo) del aprendiente, y por medio de siglas la lengua objeto (AI, para aprendiente de inglés, y AE, para aprendiente de español) y la fuente de los datos (HV, para historia de vida, y ENT, para entrevista).

\section{Resultados}

En una revisión general de los datos, sobresale que muchas de las percepciones de los aprendientes en relación con el aprendizaje de L2 se alinean con los postulados del relativismo lingüístico y con un posicionamiento desde la interculturalidad global. Es decir, se recalca la idea de que el aprendizaje de una lengua está muy ligado a nuevas y diferentes visiones de mundo y a un diálogo y comprensión entre culturas. Desde el relativismo lingüístico o la hipótesis de Sapir y Whorf se sostiene que cada lengua se asocia a diferentes formas de entender el mundo. Esta es una visión que Fanny parece compartir: "[...] me di cuenta que cada lengua abre una otra perspectiva sobre el mundo, es entender el mismo mundo de manera diferente y eso enriquece mucho el entendimiento de uno, entonces también favorece la empatía, y el amor del [sic] desconocido" (Fanny, AE-HV).

Esta forma de percibir el proceso de aprendizaje de una segunda lengua se encuentra ligada no solo a la ampliación de una cosmovisión, según lo que expresan los aprendientes, sino que también tiene que ver con la conformación de un perfil más competitivo con habilidades necesarias para la convivencia a nivel global. Charlie muestra cómo sucede esto en su narrativa: "El inglés me ha permitido comprender otras realidades más allá de las que yo vivo, pienso y siempre lo he dicho, que aprender más de una lengua te hace un ser recipiente, abierto de mente, empático y sobre todo más saludable" (Charlie, AI-HV).
Muchas de las narrativas de los participantes reflejan esta forma de pensar la cual se encaja en una visión aparentemente neutral, inocente y, por tanto, limitada de lo que involucra aprender una segunda lengua. En el campo de lenguas, sigue siendo prominente este tipo de análisis de relaciones interculturales que se ajustan a la visión de interculturalidad funcional de la que habla Walsh (2009), en la que la interculturalidad tiene que ver con el reconocimiento de la diversidad y diferencias culturales con el objetivo de alcanzar la inclusión de grupos culturales. Para este fin, son fundamentales las prácticas de interacción entre culturas basadas en el diálogo, el respeto, la convivencia y la tolerancia. Sin embargo, como lo critica Walsh, esta es una perspectiva que se acomoda a las estructuras sociopolíticas desiguales. En línea con los fundamentos teóricos de nuestro estudio y atendiendo a una perspectiva de interculturalidad crítica, Walsh aboga por el reconocimiento de la diversidad cultural con el fin de reflexionar sobre estructuras sociales e identificar las causas de desigualdades culturales, que necesitan ser combatidas.

En este contexto, desde la lente de la TCR, cuestionamos perspectivas funcionales de relaciones entre grupos sociales que encubren cuestiones de identidad permeadas por estructuras sociales jerarquizadas. Esto constituye el objetivo más importante del estudio y del análisis que presentamos en esta sección. En particular, nos referiremos a tres categorías emergentes del análisis de datos relacionadas con a) formas de identificación estratégica, b) imaginarios jerarquizados sobre lenguas y sus usuarios, y c) posibilidades de emancipación encontradas en el proceso de aprendizaje.

\section{Identificación estratégica}

Una de las maneras en que se hizo más evidente la relación entre aprendizaje de lenguas con cuestiones de identidad racial tiene que ver con diferentes formas de identificación $y$, por ende, de posicionamiento como aprendientes. El aprendizaje de una segunda lengua no muestra solamente cómo la identidad de los usuarios es transformada por este 
proceso, sino que muestra también cómo sujetos de agencia toman ventaja de este proceso para lograr estratégicamente objetivos que los favorecen dependiendo de sus condiciones y contextos (Yosso, 2005). En casos como el que se refiere Fanny en la siguiente narrativa, se puede observar que cuando el aprendiente es foráneo, podría usar esta imagen de extranjero para desafiar normas sociales de la cultura meta, sin que haya consecuencias negativas.

Pienso que de manera inconsciente es más fácil de comunicarse sobre algunos temas sensibles en otro idioma que el suyo propio, porque primero uno no se da cuenta de lo crudo o de lo tabú que es, o puede hacer caso omiso sin que sea despreciado, porque está protegido por la imagen ingenua del extranjero que habla otra lengua materna y que no sabe, la torpeza disculpada del foráneo. (Fanny, AE-HV)

Fanny deja claro que el ser extranjera en Colombia le brinda la posibilidad de estar protegida por una identidad supuestamente ingenua que le permite actuaciones comunicativas no convencionales sobre cuestiones tabú, sin ser juzgada por ello. En su caso, ser de una nacionalidad catalogada de prestigio (francesa) le otorga el privilegio de decidir si adscribirse o no a normas sociales de comunicación, que tal vez no desafiaría en su grupo cultural de origen. En otras palabras, Fanny cuenta con lo que los teóricos críticos de la raza llaman white privilege (privilegio del blanco) (Ladson-Billings y Tate, 2006) que funciona como un dispositivo por medio del cual individuos discursivamente clasificados como blancos -ya sea por su color de piel, estatus social o nacionalidad- tienen acceso a un poder simbólico o material que otros no tienen. Además, este tipo de dispositivo permite que el grupo clasificado como blanco esté libre de prejuicios (McIntosh, 2004). Como vemos, estas estructuras raciales desempeñan un papel importante en procesos de socialización en segunda lengua que se realza en determinados contextos (p. ej., en Colombia) y en determinadas situaciones (p. ej., hablar de temas tabú) con o sin implicaciones negativas (p. ej., censura).
Otra manera diferente en que el origen cultural posibilitó formas de posicionamiento ante estructuras raciales se dio por medio de lo que Spivak (1988) llama un esencialismo estratégico. En su visión poscolonialista, Spivak usa este término para referirse a situaciones en que grupos tradicionalmente marginalizados y subyugados recurren a deificar sus diferencias identitarias con otros grupos (generalmente mayoritarios) como forma de combatir dicha marginalización; dicho proceso puede ocurrir consciente o inconscientemente. Esto es precisamente lo que ocurre con Kato, aprendiente de español como segunda lengua y de ascendencia indígena. Como se observa en el siguiente fragmento de la entrevista sobre experiencias positivas de socialización en la C2, relacionada con su apariencia física y vestimenta, Kato deja claro que acentuar su identidad indígena funciona como una estrategia para visibilizar su pueblo y de reforzar percepciones positivas hacia su comunidad.

\section{E: ¿Podrías contarnos sobre una experiencia positiva relacionada con tu apariencia y ves- timenta?}

K: Pues realmente, una influencia positiva que yo podría resaltar creo que uno como persona con conocimientos, con una lengua, con una cultura tan como lo es creo que nos admiran por, sobre todo por la re-la resistencia que hemos vivido y como una cultura tan reconocida pues eso sería el-la influencia positiva.

\section{E: Tú nos hablas de una cultura reconocida, ¿en cuanto a qué?}

K: [...] una cultura reconocida me refiero a que somos una nación donde estamos organizados, a nivel económico, a nivel político y que realmente somos pueblos que, pueblos reconocidos ya a nivel ya a nivel internacional, eso no quiere decir de que somos pueblo que no tiene que ver dentro de dentro de un país, como lo es Colombia, sino que, ve venimos siendo también de ese grupo, pero que tenemos una perspectiva muy diferente a lo que tienen los demás y a eso es lo que me refiero. (Kato, AE-ENT) 
En esta narración se deja claro que la esencialización de su identidad también obedece a la celebración de la diversidad. Es decir, no solo se busca un reconocimiento político como pueblo indígena desde la diferencia, sino también un reconocimiento de la diversidad de cosmovisiones de los múltiples pueblos que habitan el territorio colombiano. En consonancia con estudios previos sobre identidad cultural y aprendizaje de lenguas (Dubeau, 2011; Valencia-Galvis, 2012), en la misma entrevista se puede ver que para Kato aprender español y educarse bajo parámetros occidentalizados de una ciudad como Bogotá cumplen un propósito instrumental para que su pueblo no quede aislado y, en la búsqueda de este objetivo, acentuar su esencia indígena se convierte en una estrategia importante de reconocimiento cultural y político.

Otro caso en el que el esencialismo estratégico cumple un rol relevante se dio en relación con otra categoría racializante: la diversidad de uso lingüístico a causa de una discapacidad auditiva. Como se explicó anteriormente, el concepto de blancura permite analizar diferentes tipos de relaciones sociales discriminatorias que, aunque no hacen referencia explícita a la raza como color de piel, se derivan de ellas y funcionan emulando las mismas jerarquías; de allí que se refiera a la racialización. Así, el caso de Lola, una aprendiente sorda, nos permite divisar estas formas de esencialización de identidad en prácticas racializadas. Aquí es posible decir que la reafirmación de una identidad esencializada parece operar en procesos de aprendizaje de esta lengua para combatir la discriminación que se ejerce hacia personas con discapacidad auditiva. Lola es una joven que desde su niñez fue perdiendo gradualmente su audición, antes de que la perdiera casi por completo, alcanzó un dominio de español oral. Así pues, como lo muestra la siguiente narrativa, su español oral ha sido el punto en consideración de la diferencia en los parámetros de lo correcto, lo organizado, lo prestigioso (lo blanco en términos poscoloniales) y esto ha sido caldo de cultivo para ponerla como objeto de burlas y actitudes racistas:
Yo decía: "Pero ¿por qué los otros me dicen que yo hablo raro, que hablo como si fuera de otro país y hacen muchas preguntas?". Algunas personas fueron muy malas conmigo, imitándome como yo hablaba imitando mi voz, hablándome y burlándose de mí, de cómo yo hablaba, entonces eso era [...] con el tema del audífono e: se ponían como a jugar a gritar para que yo mirara o para hacerme bromas muy pesadas respecto a eso porque sabían que yo tenía audífono y que eso amplificaba la voz y pues esa fue la experiencia no muy grata, la verdad no fue muy grata, en el colegio [...] conversar con los compañeros era como saber que se iban a burlar de mí, que iban a haber burlas, e:: el tema de la identidad e:: con con los profesores como que yo también sentía que me veían distinto [...]. (Lola, AE-ENT)

Para combatir y evitar este tipo de experiencias no muy gratas, Lola fue intensificando su identidad sorda, lo cual también la llevó a entender que, en su calidad de persona con discapacidad auditiva, su identidad se asimilaba a una comunidad distinta a la hispanohablante. Es decir, inició un proceso de reafirmar su identidad como sujeto perteneciente a una comunidad de habla que compartía sus mismos rasgos lingüísticos, al de la comunidad sorda, y que podría comunicarse preferencialmente mediante una lengua de señas:

[...] ya no quiero que me obliguen a hablar, a que lo diga oralmente no, no quiero porque ya estoy muy cansada de que me obliguen a hablar, fueron muchos años, treinta años, ahora soy libre ya me puedo expresar como yo me quiera expresar y eso es en la lengua de señas y en la lengua escrita prefiero. (Lola, AE-ENT)

En los tres casos discutidos en esta sección vemos cómo el aprendizaje de lenguas genera espacios de identificación diversa que favorece un posicionamiento determinado ante diversas estructuras sociales. El caso de Fanny muestra cómo el ser blanco (representar un grupo europeo) puede representar privilegios comunicacionales que otros no tienen. El caso de Kato indica cómo la esencialización de identidades podría tener fines políticos de 
representación; mientras que dicha esencialización en el caso de Lola sirve al propósito de escapar de prácticas sociales excluyentes hacia la diversidad, en particular, de capacidades lingüísticas.

Por otro lado, como veremos a continuación, los datos señalan que la identificación de individuos en términos raciales se fundamenta grandemente en imaginarios jerarquizados de las culturas relacionadas con las lenguas que están a disposición del hablante.

\section{Imaginarios jerarquizados sobre lenguas y sus hablantes}

Esta categoría surge del debate que en nuestra investigación se suscita al argumentar que las identidades raciales no pueden concebirse como categorías fijas e inflexibles que solo clasifican a individuos en etiquetas de minorías y mayorías. Los hallazgos de este estudio nos permiten extender las afirmaciones de Norton (2013), relacionadas con procesos de construcción de identidad inmersos en el aprendizaje de lenguas, y sostener que la configuración de identidad racial también es inmanente a este proceso. En nuestro análisis, lo que resulta más curioso de esta relación es que las estructuras raciales se encuentran tan inmersas en los discursos de los aprendientes que la imagen del otro y de ellos mismos está prominentemente mediada por jerarquizaciones y discursos racializados. Por ejemplo, en la siguiente narrativa se puede observar cómo Yazmín, de procedencia china, en primera instancia, mantiene una idea jerarquizada de los hablantes de español fundamentada en la visión que el marco de su cultura crea sobre lo diferente y, en consecuencia, esto la lleva a verse a sí misma en un proceso de transformación que surge mediante el aprendizaje del español.

Personalmente, personas que hablan demasiado rápido como españoles no me parecen tranquilos ni elegantes, porque en China no se considera respetuoso sino un poco precipitado y ofensivo hablar excesivamente rápido [...]
[Con respecto a mi experiencia en Colombia] me di cuenta de que me había vuelto más optimista y sentía mayor confianza al conversar con la gente. Esto también es lo que me comentan mis amigas quien dicen que ya no soy tan china (los chinos son más tímidos, conservadores y callados). Supongo que es por la influencia que me traen el idioma, la cultura y la gente hispana. (Yazmin, AE-HV)

Aunque, como se afirmó anteriormente, este imaginario sobre quiénes son los españoles se deriva de la forma como su cultura china ha influido en sus formas de concebir lo diferente (Brislin, 1993), es posible ver en la segunda parte de su narrativa que, como lo dirían los críticos de la raza, la construcción que hace de ella misma se adhiere a discursos globales dominantes de exotización de las culturas latinas (Delgado y Stefancic, 2001). Esto tiene que ver con la racialización de los discursos a la que se hacía referencia y que se derivan de un dispositivo de blancura (Castro-Gómez, 2005) en los que se ha perpetuado la imagen de perfección al idealizado blanco (europeo, intelectual) mientras que el otro (en este caso latino hispanohablante) se ve como diferente y con características que cimientan la idea arraigada en lo afectivo y sensible, pero que en últimas no llegan a equipararse con la bondad e ilustración del blanco (por lo general, europeo) (Mignolo, 2000; CastroGómez, 2000). En este caso, a pesar de que Yazmín utiliza una narrativa optimista de afiliación con los hispanohablantes latinos a través de su experiencia en el contexto colombiano, este extracto también refleja esta adherencia explicada en oposición a lo serio, aplomado y educado (lo blanco) que forma parte de discursos globales dominantes que sitúan al blanco en el centro de la intelectualidad, mientras que los otros poseen capacidades que generalmente tienen que ver con lo pasional o sensible, pero no lo intelectual.

Una extensión de esta categoría muestra que incluso la identidad de aprendientes de segundas lenguas es todavía más inestable, flexible y fluida en la forma como una lengua se convierte en aquella dimensión que pareciera permitirle al usuario 
posicionarse de manera diferente en situaciones comunicativas, dependiendo de los significados que le otorgan a cada lengua. Fanny nos muestra cómo sucede esto:

El español me parece una lengua muy hermosa y se presta mucho para expresar pasiones y sentimientos, lo siento así porque cuando puedo elegir (que hablo a una persona que también habla francés y español), elijo el español para lo cariñoso y el francés para filosofar y pensar. (Fanny, AE-HV)

Al igual que sucede con Yazmín, Fanny deja ver que sus apreciaciones sobre la lengua tienen que ver con las ideas jerarquizadas en los discursos racializados sobre el valor sociocultural desigual de esas lenguas. En el caso del español, hace alusión al polo de la sensibilidad en contraste con el francés referenciando a lo intelectual. Es decir, implícitamente se establece una relación entre la comunidad lingüística francesa con el privilegiado mundo del blanco, mientras que la comunidad lingüística española parece representar su opuesto minorizado, que puestos desde la teoría de la blancura simbolizarían el negro, mestizo, latino o diferente, colonizado (Castro-Gómez, 2000).

Hasta este punto hemos discutido cómo el aprendizaje de lenguas se entrelaza con diferentes formas de autoentendimiento e identificación que lleva a posicionar a los individuos de maneras diversas en el entramado de estructuras sociales inequitativas. A continuación, veremos cómo el aprendizaje de lenguas también representa oportunidades de resignificación de dichas estructuras.

\section{Posibilidades de emancipación}

La conexión entre identidad racial y aprendizaje de lenguas se ve complejizada en este estudio de manera interesante al encontrar que, desde el punto de vista de los aprendientes, las lenguas han servido de puentes para buscar sacudirse de estructuras sociales opresoras y entender su realidad de manera diferente (Carbado y Gulati, 2003, Yosso, 2005). En este caso, entendiendo desde la TCR que nos encontramos inmersos en unas estructuras de poder que han enmarcado prácticas sociales desde la centralidad de la blancura (p. ej., lo estándar, lo socialmente más aceptado y deseado) y con esta se configuran discursos racializados (Castro-Gómez, 2000; Mignolo, 2000). Las posibilidades de emancipación de dichas estructuras germinan cuando el aprendizaje de una segunda lengua representa una forma diferente de entender la realidad social. En este estudio, observamos que los aprendientes logran identificar y desafiar estructuras sociales relacionadas con orientación sexual y roles de género.

En cuanto al primer aspecto, tenemos el caso de Charlie, un aprendiente de inglés quien nos presenta una narrativa en la que se hace visible cómo su experiencia de aprender una segunda lengua lo ha llevado a escabullirse de las estructuras sociales que enmarcan el ideal del hombre blanco, intelectual $y$ heterosexual en el contexto donde se encuentra inmerso.

El inglés me ha permitido comprender otras realidades más allá de las que yo vivo [...]. He sido capaz de conectarme con algo que por mucho tiempo no era completamente consciente y era que yo pertenezco a la comunidad LGBT + y me identifico no género binario con orientación homosexual. Poder experimentar mediante un nuevo idioma experiencias de vida de otras personas que lucharon y han luchado por conseguir ser visibles en una sociedad injusta es acogedor $y$ motivante. (Charlie, AI-HV)

De acuerdo con Beltrán-Cely (2013), el contexto colombiano, a pesar de haberse transformado, conserva una visión tradicional de familia en que la sexualidad es racializada, es decir, fundamentada en la heterosexualidad, discriminando lo diferente. Para Charlie, aprender inglés ha significado tener la posibilidad de posicionarse más abiertamente como una persona homosexual. Charlie muestra que ser aprendiente de inglés le ha permitido encontrarse con otras personas de otras culturas que podían expresar su homosexualidad de manera menos restringida que la de su contexto. También le ha permitido entender que esto ha sido producto de luchas sociales. 
Aprender una segunda lengua le ha brindado a Charlie posibilidades de viajar a otros contextos $y$, aunque no lo ha hecho de manera presencial, en la entrevista expresa cómo interacciones virtuales a través de internet con personas angloparlantes de otros contextos le ha servido para aprender que la segregación en la que se hallaba inmerso era no solo una problemática que otros también enfrentaban, sino que estas personas luchaban por defender cómo se definían. Esto llevó a Charlie a darse cuenta de que el inglés podría ser una forma de cambiar ese acomodamiento a la estructura racial fundamentada en el blanco, heterosexual y ponerlo en una posición de lucha y confrontación con quien él era.

Otro caso de posibilidades de emancipación mediadas por el aprendizaje de una segunda lengua lo presenta Mona, aprendiente de español procedente de la India, quien haciendo referencia a su contexto natal expresa que aprender español, específicamente en Colombia, ha sido una herramienta para conocer un contexto externo que la ha ayudado a entenderse a como mujer de manera diferente:

Ser mujer en la India es algo de lo que eres muy consciente [...] siempre eres consciente de que eres una mujer, de alguna manera la estructura social es de esa manera, para vestirte de alguna manera tienes que pensar en ello, así que necesitas pensar bien cómo vestirte, ah tienes que pensar bien cómo hablar con la gente, cuando caminas en la calle debes ser consciente del hecho de que eres una mujer, [...] esta es una sociedad es muy patriarcal y muy estricta, eres consciente de ello todo el tiempo y aquí [en Colombia] no es así, creo que si regreso a la India eso sería lo que sería el choque cultural inverso [risas] ese sería el punto de que si voy a la India [...] no me gustaría eso, que yo estoy todo el tiempo consciente del hecho de que soy una mujer. (Mona, AE-ENT)

Desde el punto de vista específicamente del contexto de hablantes, Mona muestra cómo aprender una segunda lengua, en este caso el español de Colombia, la ayudó a liberarse de algunas estructuras culturales relacionadas con los roles de género en su contexto indio y, por tanto, entender que la cultura es un factor determinante para la forma en que se cree que se debe asumir el rol de la mujer. En este sentido, la TCR discute los discursos racializados que parten de la blancura (reflejada aquí en los parámetros de comportamientos aceptados) para organizar el mundo social. Estos discursos tienen que ver con las prácticas sociales que se intersecan con otras categorías y van mucho más allá del color de piel y que discriminan teniendo parámetros de idealización en el individuo blanco, heterosexual, masculino (Carbado y Gulati, 2003). La narración de Mona muestra que aprender español en el contexto colombiano representó para ella la concienciación de que la racialización de las prácticas sociales alrededor del rol femenino en la India es marcada y que determina prototipos específicos de ideal femenino, que como lo dirían Castro-Gómez (2000) y Mignolo (2000), deben encajar con el dispositivo de blancura.

Teniendo en cuenta cómo Charlie y Mona recurren a narrativas que muestran la complejidad en que se dan los discursos racializados y de su intersección con otras categorías, como la de género, es posible afirmar que analizando estas relaciones se posibilitan no solo nuevas formas de ver la discriminación y la exclusión, sino también de ver la emancipación, dadas por la creatividad de los individuos y los saberes a los que tienen acceso, en este caso, al ser usuarios de una segunda lengua.

\section{Conclusiones}

En términos generales, analizar el aprendizaje de lenguas desde la lente de la TCR -que toma en cuenta las dimensiones históricas y estructurales de coexistencia e interrelación de grupos socialespermite visibilizar relaciones de poder de tipo social, político y cultural que han mediado la configuración de percepciones que se pueden llegar a tener sobre determinados grupos sociales y comunidades de habla. Como lo muestra este estudio, dichas relaciones de poder y percepciones forman parte inherente del proceso de aprendizaje de una segunda lengua. Por tanto, desarrollar este tipo de análisis permite tratar las cuestiones de raza mucho más allá del frecuente énfasis meramente en el color de piel. Como se ha dilucidado, esta perspectiva teórica 
toma la raza como una dimensión simbólica desde donde es posible examinar y cuestionar otro tipo de jerarquizaciones y desigualdades sociales que pueden estar relacionadas explícita o implícitamente con la raza.

Teniendo en cuenta este piso teórico, uno de los mensajes más importantes que nos deja el presente estudio es que la relación entre el aprendizaje de lenguas e identidad racial tampoco se limita a lo que comúnmente se ha denominado comunidades minoritarias. Desde los cimientos de la TCR, como se ha mostrado en este análisis, cualquier aprendiente de segundas lenguas es susceptible de negociar su identidad, la cual está mediada por estructuras raciales que complejizan las prácticas sociales. La naturaleza de dicha negociación puede tomar diversos caminos dependiendo primordialmente del grado de acomodación o cuestionamiento de dichas estructuras. Este estudio muestra que algunos aprendientes, en particular los provenientes de contextos reconocidos como de prestigio, pueden gozar de un privilegio de blancura que les permite utilizar su identidad para no ser objeto de juicios de valor negativos hacia prácticas comunicativas censurables dentro de un grupo cultural específico. Por otro lado, cuando se trata de aprendientes provenientes de comunidades a las que se les ha otorgado menos prestigio (en este caso: indígenas o sordos), los aprendientes buscarían cuestionar estructuras sociales desfavorables por medio de la esencialización de algunos de sus aspectos identitarios.

Gracias a la lente de la TCR, es posible determinar que, dentro de la línea de acomodación a estructuras racializadas, las identificaciones que se pueden llegar a hacer hacia otros grupos sociales se fundamentan en gran medida en ideas jerarquizadas y muchas veces estereotipadas que provienen de discursos globales dominantes. Por ejemplo, las caracterizaciones que los aprendientes hacen sobre el español (y sus hablantes) se vieron frecuentemente asociadas con el plano emocional (esto es, extroversión, alegría, sensibilidad). Estas asociaciones contrastan con ideas sobre otros grupos linguoculturales más asociados con intelectualismo, como es el caso del francés.
Los hallazgos presentados aquí representan potencial para consideraciones en la enseñanza y aprendizaje de segundas lenguas que, según lo reportado en los estudios realizados a la fecha, fundamentalmente se han centrado en la esencialización de identidades raciales, culturales y étnicas, generadas desde los discursos dominantes que perpetúan relaciones de poder en desigualdad. Muchos de esos discursos parecen ser mayormente distribuidos desde políticas educativas y medios de comunicación: La circulación de estos discursos tiende a ignorar identidades transitorias que reflejan unos hablantes reales situados en el mundo social que toman ventaja de las herramientas y saberes que poseen para ser agentes de cambio. Esta creatividad dada por los hablantes de acuerdo con sus contextos es un elemento que necesita atención pedagógica para poder abordar prácticas que no solo propendan por experiencias de aprendizaje más reales, sino que también promuevan la construcción de una sociedad más igualitaria para lo cual se vuelve necesario entender cómo las estructuras sociales inequitativas pueden ser reproducidas, pero también, y sobre todo, combatidas en procesos de enseñanza y aprendizaje de lenguas.

\section{Referencias}

Agudelo, J. J. (2007). An intercultural approach for language teaching: developing critical cultural awareness. Ikala, 12(18), 185-217.

Alim, S., Rickford, J. y Ball, A. (2016). Raciolinguistics: how language shapes our ideas about race. Oxford University Press.

Anderson, K. (2015). Racializing language: unpacking linguistic approaches to attitudes about race and speech. En J. Bloomquist, L. J. Green y S. L. Lanehart (eds.), The Oxford Handbook of African American Language (pp. 771-775). Oxford University Press.

Anya, U. (2017). Racialized identities in second language learning: speaking blackness in Brazil. Routledge.

Arreguín-Anderson, M. G. y Ruiz-Escalante, J. A. (2014). Una perspectiva crítica racial de la opresión lingüística desde el lente de voces chicanas. Journal of Latinos and Education, 13(1), 54-61. https//doi. org/10.1080/15348431.2013.800814 
Banks, J. A. (2002). Race, knowledge construction, and education in the USA: lessons from history. Race, Ethnicity and Education, 5(1), 7-27. https//doi. org/10.1080/13613320120117171

Banton, M. (2002). Racial theories. Cambridge University Press.

Bello, M. y Rangel, M. (2000). Etnicidad, “raza” y equidad en América Latina y el Caribe. Revista de Cepal, 76(4), 39-54.

Beltran-Cely, W. (2013). Pluralización religiosa y cambio social en Colombia. Theologica Xaveriana, 63(175), 57-85.

Bonilla-Medina, S. X. (2018). Racial identity in educational practices in the context of Colombia. [Tesis sin publicar]. University of East London.

Bonnet, A. (2000). White identities: historical and international perspectives. Prentice Hall.

Brislin, R. (1993). Understanding culture's influence on behavior. East West Honolulu Center.

Carbado, D. W. y Gulati, M. (2003). The law and economics of critical race theory (Book Review). Yale Law Journal, 12, 1787-1828.

Castro-Gómez, S. (2000). Ciencias sociales, violencia epistémica y el problema de la invención del otro. En E. Lander (ed.), La colonialidad del saber, eurocentrismo y ciencias sociales (pp. 145-163). Clacso.

Castro-Gómez, S. (2005). La hybris del punto cero: ciencia, raza e ilustración en la Nueva Granada (1750-1816). Pontificia Universidad Javeriana.

Clarke, S. y Garner, S. (2009). White identities: a critical sociological approach. Pluto Press.

Clavijo, A (2017). Voces invisibles de las minorías indígenas en Colombia bilingüe. En E. Mateus y A. Tonelli (eds.), Diálogos (im)pertinentes entre formação de professores e aprendizagem de línguas (pp. 163-177). Blucher.

Cuba-Vega, L. (2014). Enseñanza del español e identidad afrodescendiente. Index. Comunicación: Revista Científica en el Ámbito de la Comunicación Aplicada, 4(2), 139-158.

Chadderton, C. (2009). Discourses of britishness, race and difference: minority ethnic students' shifting perceptions of their school experience. [Tesis de doctorado]. The Manchester Metropolitan University.

Chaves, M. y Zambrano, M. (2006). From blanqueamiento to reindigenización: Paradoxes of mestizaje and multiculturalism in contemporary Colombia. Revista Europea de Estudios Latinoamericanos y del Caribe, 80, 5-23.

Davies, B. y Harré, R. (1990). Positioning: the discursive production of selves. Journal for the Theory of Social Behaviour, 20(1), 43-63.

DeCuir, J. T. y Dixson, A. D. (2004). "So, when it comes out, they aren't that surprised that it is there": using critical race theory as a tool of analysis of race and racism in education. Educational Researcher, 33(5), 26-31.

De Fina, A. y Georgakopoulou, A. (2012). Analyzing narrative: discourse and sociolinguistic perspectives. Cambridge University Press.

Delgado, R. y Stefancic, J. (2000). Critical race theory: the cutting edge. Temple University Press.

Delgado, R. y Stefancic, J. (2001). Critical race theory an introduction. New York University Press.

Du Bois, B. (2001). The conservations of races. En R. Bernasconi (ed.), Race (pp. 84-92). Blackwell Publishers.

Dubeau, G. (2011). La identidad autóctona y el aprendizaje del español como segunda lengua en comunidades indígenas de Hispanoamérica: el caso de Ecuador. [Tesis de maestría]. Universidad de Montreal.

Esin, C. (2011). Narrative analysis approaches. En N. Frost (ed.), Qualitative Research methods in psychology (pp. 92-118). McGraw-Hill.

Fanon, F. (2001). The lived experience of the black. En R. Bernasconi (ed.), Race (pp. 184-202). Blackwell Publishers.

Flórez-Bolívar, F. J. (2008). Representaciones del Caribe colombiano en el marco de los debates sobre la degeneración de las razas: geografía, razana y nación a comienzos del siglo xx. Historia y Espacio, 4(31), 35-61.

Gillborn, D. (2010). Reform, racism and the centrality of whiteness: Assessment, ability and the 'new eugenics'. Irish Educational Studies, 29(3), 231-252. https//doi.org/10.1080/03323315.2010.498280

Gilroy, P. (2004). Between camps: nations, cultures and the allure of race. Penguin Books.

Harré, R., Moghaddam, F. M., Cairnie, T. P., Rothbart, D. y Sabat, S. R. (2009). Recent advances in positioning theory. Theory \& Psychology, 19(1), 5-31. https// doi.org/https://doi.org/10.1177/0959354308101417 
King, J. (1991). Dysconscious racism: ideology, identity, and the miseducation of teachers. The Journal of Negro Education, 60(2), 133-146.

Knowles, C. y Alexander, C. (2005). Making race matter: bodies, space and identity. Palgrave Macmillan.

Koopman, S. (2012). "Mona, mona, mona!" Whiteness, tropicality, and international accompaniment in Colombia. Paper presented at the SFU/UBC Latin American Studies Working Paper Series, Vancouver. http://summit.sfu.ca/item/10695

Kubota, R. y Lin, A. (2009). Race, culture, and identities in second language education: introduction to research and practice. En R. Kubota y A. Lin (eds.), Race, culture and identities in second language education: exploring critically engaged practice (pp. 1-24). Routledge.

Ladson-Billings, G. y Tate, W. F. (2006). Education research in the public interest: social justice, action, and policy. Teachers College Press.

Lefèvre, S. y Okome-Beka, V. (2015). Invisibilidad, visibilidad y "mal-visibilidad" de las poblaciones afro en los libros de enseñanza de español como lengua extranjera (ELE). Una mirada cruzada entre Francia y Gabón. Matices en Lenguas Extranjeras, (9), 47-67. https://doi.org/10.15446/male.n9.54912

Leonardo, Z. (2002). The souls of white folk: critical pedagogy, whiteness studies, and globalization discourse. Race, Ethnicity and Education, 5(1), 29-50. https//doi. org/10.1080/13613320120117180

Leung, C., Harris, R. y Rampton, B. 1997. The idealised native speaker, reified ethnicities, and classroom realities. TESOL Quarterly, 31(3), 543-560. https// doi.org/10.1080/13613320120117180

McGraw, J. (2007). Purificar la nación: eugenesia, higiene y renovación moral-racial de la periferia del Caribe colombiano, 1900-1930. Revista de Estudios Sociales, (27), 62-75.

McIntosh, P. (2004). White privilege: Unpacking the invisible knapsack. Race, Class, and Gender in the United States, 6, 188-192.

Mignolo, W. (2000). La colonialidad a lo largo y a lo ancho: el hemisferio occidental en el horizonte colonial de la modernidad. En E. Lander (ed.), $L a$ colonialidad del saber: eurocentrismo y ciencias sociales. Perspectivas latinoamericanas (pp. 55-86). Clacso.

Mignolo, W. (2005). Cambiando las éticas y las políticas del conocimiento: lógica de la colonialidad y postcolonialidad imperial. Tabula Rasa, 3, 47-72.
Ministerio de Educación Nacional (MEN). (2013). Orientaciones para la implementación de proyectos de fortalecimiento de inglés en las entidades territoriales. Bogotá. http://www.colombiaaprende.edu.co/ lenguasextranjeras/www.mineducacion.gov.co

Ministerio de Educación Nacional (MEN). (2014). Programa Nacional de Inglés 2015-2025: documento de socialización. Bogotá. http://www.colombiaaprende.edu.co/html/micrositios/1752/articles343287_recurso_1.pdf

Ministerio de Educación Nacional (MEN). (2016). Colombia bilingüe. Bogotá. http://aprende.colombiaaprende.edu.co/es/colombiabilingue/86689

Ministerio de Cultura (23 de marzo de 2018). Colombia, destino por excelencia para aprender español. Bogotá. https://www.mincultura.gov.co/prensa/noticias/ Paginas/Colombia,-el-destino-por-excelencia-paraaprender-espa\%C3\%B1ol-.aspx

Morgan, B. (2004). Teacher identity as pedagogy: towards a field-internal conceptualisation in bilingual and second language education. International Journal of Bilingual Education and Bilingualism, 7(2-3), 172-188. https//doi.org/10.1080/13670050408667807

Muñoz-Gaviria, D. A. y Runge-Peña, A. K. (2005). El evolucionismo social, los problemas de la raza y la educación en Colombia, primera mitad del siglo xx: el cuerpo en las estrategias eugenésicas de línea dura y de línea blanda. Revista Iberoamericana de Educación, (39), 127-168.

Nardal, P. (2001). The awakening of race consciousness. En R. Bernasconi (ed.), Race (pp. 107-113). Blackwell Publishers.

Norton, B. (1997). Language, identity and the ownership of English. TESOL Quarterly, 3(3), 409-429.

Norton, B. (2013). Identity and language learning: extending the conversation. Multilingual Matters.

Ospina, W. (1997). Colombia, el proyecto nacional y la franja amarilla. Editorial Norma.

Quiceno, H. (2003). Crónicas históricas de la educación en Colombia. Cooperativa Editorial Magisterio, Universidad Pedagógica Nacional.

Redondo-Quesada, B. (2018). La dualidad de la identidad hispanounidense en la enseñanza de español para hispanohablantes en los institutos de los Estados Unidos. [Tesis de maestría sin publicar]. Universidad de Barcelona. 
Rosa, J. y Flores, N. (2017). Unsettling race and language: toward a raciolinguistic perspective. Language in Society, 46(5), 621-647. https//doi.org/10.1017/ S0047404517000562

Said, E. W. (1976). Orientalism. Penguin Books.

Sleeter, C. E. (2004). How white teachers construct race. En D. Gillborn y G. Ladson-Billings (eds.), The Routledge Falmer reader in multicultural education (pp. 163-178). Routledge Falmer.

Soler, C. S. (2006). Racismo discursivo de élite en los textos escolares deficiencias sociales en Colombia1. Revista de Investigación, 6(2), 255-260.

Solorzano, D. G. y Yosso, T. J. (2001). Maintaining social justice hopes within academic realities: a freirean approach to critical race/latcrit pedagogy. Denver Law Review, 78(4), 595-621.

Somers, M. R. (1994). The narrative constitution of identity: a relational and network approach. Theory and Society, 23(5), 605-649.

Spector-Mersel, G. (2010). Narrative research: time for a paradigm. Narrative Inquiry, 20(1), 204-224. https:// doi.org/10.1075/ni.20.1.10spe

Spivak, G. C. (1988). Can the subaltern speak? En R. Morris (ed.), Can the subaltern speak? Reflections on the history of an idea (pp. 1-21). Columbia University Press.

Thesen, L. (1997). Voices, discourse and transition. In search of new categories in EAP. TESOL Quarterly, 31(3), 487-511.

Usma, J., Medina, J. y Ortiz, C. (2018). Indigenous students learning English in higher education: challenges and hopes. Ikala, Revista de Lenguaje y Cultura, 23(2), 229-254. http://dx.doi.org/10.17533/ udea.ikala.v23n02a03

Valencia-Galvis, R. (2012). Comunidad quechua en Medellín: lengua, identidad y cultura. Boletín de Antropología, 27(44), 304-318.

Wade, P. (1995). Blackness and race mixture: the dynamics of racial identity in Colombia. JHU Press.

Walsh, C. (2009). Interculturalidad crítica y pedagogía de-colonial: apuestas (des)de el in-surgir, re-existir y re-vivir. UMSA Revista (entre palabras), 3, 1-30.

Yosso, T. J. (2005). Whose culture has capital? A critical race theory discussion of community cultural wealth. Race Ethnicity and Education, 8(1), 69-91. https//doi. org/10.1080/1361332052000341006 